\title{
Antiretroviral Therapy through Barriers: A Prominent Role for Nanotechnology in HIV-1 Eradication from Sanctuaries
}

\author{
Fabio Corsi ${ }^{1}$, Luca Sorrentino ${ }^{1}$, Serena Mazzucchelli ${ }^{1}$, Marta Truffi ${ }^{1}$, Amedeo Capetti ${ }^{2}$, Giuliano Rizzardini ${ }^{2,3}$ and \\ Luisa Fiandra ${ }^{1}$ \\ 1. Università degli Studi di Milano, Dipartimento di Scienze Biomediche e Cliniche “L. Sacco”, via G.B. Grassi 74, 20157, Milan, \\ Italia \\ 2. Ospedale L. Sacco, via G.B. Grassi 74, 20157, Milan, Italia \\ 3. University of the Witwatersrand, Faculty of Health Science, School of Clinical Medicine, 7 York Rd \& Carse O'Gowrie Road, \\ Johannesburg, South Africa
}

\begin{abstract}
In HIV-1 management, eradication of the virus from sanctuaries represents a major and challenging goal. The genital tract, gut associated lymphoid tissue, lymph nodes, central nervous system, macrophages and latently infected CD4+ T lymphocytes are typical sites where HIV-1 compartmentalizes. To circumvent this problem, a consistent number of studies have focused on improving ARVs (antiretroviral drugs) delivery into sanctuary sites and different nanotechnological approaches have been developed. Cellular HIV-1 sanctuaries (i.e. macrophages) can be reached by nanoformulation of ARVs or by activation of latently infected cells. Anatomical sanctuaries (i.e. brain or male genital tract) can be addressed by increasing the permeation of ARVs across tissue barriers, such as the blood-brain barrier or the blood-testis barrier, while ARVs concentration in lymph nodes can be enhanced by drug encapsulation in CD4-targeted nanoparticles.
\end{abstract}

Key words: Nanotechnology, HIV-1, antiretrovirals, sanctuaries, delivery.

\section{Introduction}

In the era of tailored cure and global progression of medicine in most fields, eradication of HIV-1 infection is still a clinical challenge. Although current ARV (antiretroviral) therapies reduce plasma HIV-1 below detectable levels in a consistent proportion of subjects [1], to date, a complete viral eradication is unfeasible. Even a five drugs-regimen in early $(<6$ months) HIV-1 infection, which represents the strongest therapy in the best HIV-1 setting, does not improve the reservoir reduction in terms of proviral DNA, cell-associated RNA, replication-competent virus (all from purified blood CD4+ T cells), or gut

Corresponding author: Luisa Fiandra, Ph.D., Università degli Studi di Milano, Dipartimento di Scienze Biomediche e Cliniche “L. Sacco”, via G.B. Grassi 74, 20157, Milan, Italia. proviral DNA [2]. The major cause of failure to eradicate is the persistence of the virus in certain physiological reservoirs. These HS (HIV-1 sanctuaries) can be defined as anatomical (i.e. central nervous system, genital tract, gut-associated lymphoid tissue, lymph nodes) or cellular (i.e. latently infected CD4+ T cells, macrophages) protected sites, which are almost impermeable to ARVs action and in which HIV-1 is able to replicate despite treatments, thus preventing any possibility of cure [3]. Nowadays, a combination of ARVs that target multiple steps of HIV-1 replication (highly active antiretroviral therapy, HAART), represents the standard of care for HIV-1 infection. However all these drugs belong to different classes with different pharmacokinetic profiles.

In this scenario, nanotechnology for HIV-1 therapy is an exciting field of research, since: (1) 
nanoformulation may significantly improve the bioavailability and pharmacokinetic profile of ARVs allowing them to penetrate into HS for a proper eradication of the virus; (2) nanoparticles are efficient drug delivery systems, which could combine several ARVs, thus providing a real hope for HIV-1 cure. Nanotechnological strategies for HIV-1 therapy have been investigated for more than 20 years. The first evidence that nanotechnology can improve ARV delivery comes from an experimental study in 1992, a few years after HIV-1 discovery. Polyalkylcyanoacrylate nanoparticles were found to be promptly phagocytized by HIV-1-infected macrophages in vitro, promoting the incorporation of delivered azidothymidine into the target cells [4]. Since that study, a significant part of the nano-research has focused on improving the bioavailability of ART (antiretroviral therapy). Since 2009, Gendelman and colleagues have developed the so-called "nanoART", a polymer excipient-coated drug nanosuspension with high drug loading capacity, controllable size and charge, and tunable surface conjugation [5, 6]. NanoART demonstrated a high efficacy in controlling HIV-1 infection, with more than 1000-fold reduction in viral load upon weekly injections in murine models [7]. This nanoparticle can be efficiently internalized into monocyte-derived macrophages, acting as delivery systems of ARVs to these HIV-1 reservoirs, and the ability of macrophages to take in and release nanoART is associated with an improved antiretroviral efficacy in infected cells $[8,9]$.

Although the role of nanotechnology in improving ARV's bioavailability to infected cells has been widely described, overcoming HIV-1 sanctuaries remains a great challenge. Indeed, the contribution of nanoformulation in "shuttling" ARVs into HS is still poorly investigated. With regard to the CNS (central nervous system), nanoparticles that are able to improve drug permeation across the BBB (blood brain barrier) could allow ARVs to reach brain HIV-1-sensitive CD4+ cells. This goal could provide an exciting new perspective on HIV-1 therapy, since 98\% of ARVs are not able to reach this HIV-1 reservoir [10]. Overcoming the BTB (blood-testis barrier) would similarly aid in the penetration of ARVs into the MGT (male genital tract). In the lymph nodes, where the optimal ARV concentration is unknown, the challenges presented by the presence of different types of cellular reservoirs should be faced by conjugating ARVs to anti-CD4-targeted nanoparticles.

Purpose of this review is to describe HIV-1 sanctuaries and the principal biological barriers hindering delivery of ARVs, and to provide an overview of the nanotechnological approaches implemented to properly address ARV delivery to HIV-1 reservoirs. The accomplishment of these studies will likely have a strong clinical impact on HIV-1 management, increasing the efficacy of ARVs and limiting their side effects.

\section{HIV-1 Sanctuaries}

2.1 Anatomical Sites: Central Nervous System, Male Genital Tract and Lymph Nodes

CNS is considered one of the most important anatomical sanctuaries for HIV-1. It displays an elevated concentration of infected macrophages and microglial cells, which promote inflammatory escalation and lead to astrogliosis and neurodegeneration $[3,11]$. The low permeability of $\mathrm{BBB}$ is responsible for the exclusion of vast majority of antiretroviral drugs from CNS. The BBB is composed of a layer of brain microvascular endothelial cells, reinforced by pericytes and astrocytes endfeet, which strictly prevent the access to all the hydrosoluble drugs with a molecular weight higher than 400 Da. Molecules unable to cross the BBB because of their size, weight or polarity, can cross the BBB only if transported by receptor-mediated transcytosis. Indeed, the cerebrospinal fluid has been demonstrated to be inaccessible to most ARVs, such as nelfinavir, 
ritonavir [12], and saquinavir [13], as well as the nucleotide analogue tenofovir [14] and the fusion inhibitor enfuvirtide [15]. This fact is clinically relevant, since HIV-1-related brain infection leads to a broad spectrum of complex neurocognitive and organic disorders, altogether called "neuroAIDS" [10]. It includes cognitive decline with impairment of attention, learning and mood disorders up to dementia, and other manifestations such as multifocal leukoencephalopathy, primary CNS lymphoma and tuberculous or cryptococcal meningitis [16]. The advent of ART has drastically reduced the most severe forms of neuroAIDS, but the prevalence of milder neurologic signs (e.g., asymptomatic neurocognitive impairment and mild neurocognitive impairment) remains high, affecting up to half of all HIV-1-infected individuals [17] and conferring a 2- to 6-fold increased risk for the development of symptomatic HIV-1-associated neurocognitive disorder [18]. In the ART-naive population, neurocognitive impairment has been associated with higher HIV-1 RNA and DNA levels in plasma and cerebrospinal fluid, and with several markers of inflammation and monocyte activation [19, 20]. A huge amount of HIV-1 DNA in the peripheral blood has been associated with severe neurocognitive impairment, independently of HIV-1 RNA plasma load [21]. In the setting of virologic suppression following ART, greater HIV-1 DNA levels contribute to persistent immune activation in tissues, including the brain [22], which in turn contributes to neurodegeneration and cognitive impairment [23]. Finally, there is evidence that the cerebrospinal pool of HIV-1 is one of the major obstacles to eradication [24] and therefore strategies to make ARVs overcome the BBB are urgently needed.

Another anatomical barrier to the ARVs is represented by the blood-testis barrier, one of the tightest blood-tissue barriers in mammalians. It separates an apical compartment, where meiosis, spermiogenesis and spermiation occur, from a basal compartment, where the spermatogonial differentiation and the development up to the preleptotene spermatocyte stage take place. BTB is a dynamic barrier: a new BTB is produced behind the transiting spermatocytes, while the previous layer undergoes degeneration above the cells in transit [25]. The tightness of the BTB is due to specialized tight junctions between the Sertoli cells, which hamper the intercellular diffusion of hydrophilic molecules. The penetration of ARVs into the MGT, expressed as the ratio of drug concentration in semen to plasma samples, was analysed by Else and collegues on a wide variety of ARVs belonging to different classes [26]. The rank order of accumulation is reported in Table 1. The BTB has a high clinical significance since HIV-1 is sexually transmitted. In most cases, although viral load is completely depleted from blood,

Table 1 Rank order of ARV accumulation into the MGT (male genital tract).

\begin{tabular}{|c|c|c|}
\hline Rank order & ARV & Class \\
\hline \multirow[t]{4}{*}{1} & Lamivudine & NRTIs \\
\hline & Zidovudine & NRTIs \\
\hline & Tenofir & NRTIs \\
\hline & Didanosine & NRTIs \\
\hline \multirow[t]{2}{*}{2} & Stavudine & INSTIs \\
\hline & abacavir & PIs \\
\hline 3 & raltegravir & EIs \\
\hline \multirow[t]{3}{*}{4} & indinavir & PIs \\
\hline & maraviroc & EIs \\
\hline & nevirapine & NNRTIs \\
\hline \multirow[t]{4}{*}{5} & efavirenz & NNRTIs \\
\hline & amprenavir & PIs \\
\hline & atazanavir & PIs \\
\hline & darunavir & PIs \\
\hline \multirow[t]{2}{*}{6} & lopinavir & PIs \\
\hline & ritonavir & PIs \\
\hline 7 & saquinavir & PIs \\
\hline 8 & enfuvirtide & FIs \\
\hline
\end{tabular}

NRTIs: nucleoside/nucleotide reverse transcriptase inhibitors; NNRTIs: non-nucleoside reverse transcriptase inhibitors; INSTIs: integrase strand transfer inhibitors; PIs: protease inhibitors; EIs: entry inhibitors; FIs: fusion inhibitors. 
HIV-1 remains in semen [27]. Reasons of such HIV-1 persistence are still to be investigated in detail, but it seems that both the BTB and the presence of HIV-1-infected macrophages in urethra and other male genital organs could be responsible for active release of HIV-1 in semen of patients undergoing HAART, thereby increasing the probability of HIV-1 transmission in sexually active patients [28, 29].

Despite the effectiveness of ART, HIV-1 remains detectable in lymph nodes [30], and an insufficient exposure of lymphoid tissue to ARVs is a crucial aspect with regards to complete viral eradication. Indeed, Kinman et al. demonstrated that indinavir concentration in lymph node mononuclear cells was about one third of that found in similar cells within the blood in HIV-1-positive patients [29]. Moreover, a recent study on 12 HIV-1-infected patients treated over six months with five of the most frequently used ARVs [tenofovir disoproxil fumarate/emtricitabine, in combination with efavirenz ( $\mathrm{n}=6)$, atazanavir/ritonavir $(\mathrm{n}=4)$, and darunavir/ritonavir ( $\mathrm{n}$ =2)], demonstrated that concentration of the drugs in lymph nodes was much lower than that detected in the peripheral blood [31].

\subsection{Cellular Sites}

Lymphatic tissues can be considered HS not only for the poor accessibility to ARVs, but also for the abundant presence of major cellular reservoirs in these sites. A study on SIV-infected macaques, treated with a combined antiretroviral therapy for 1 year, showed that viral RNA was undetectable in plasma, while remained detectable in productively infected $\mathrm{T}$ lymphocytes. These cells were predominantly found clustered in the follicles of mesenteric lymph nodes, and were responsible for the rebound of plasma viremia upon cessation of therapy [32].

However, the main cellular reservoirs for latent HIV-1 infection are the central memory $\left(\mathrm{T}_{\mathrm{CM}}\right)$ and the transitional memory $\left(\mathrm{T}_{\mathrm{TM}}\right) \mathrm{CD} 4+\mathrm{T}$ cells. In patients subjected to early HAART treatment, $\mathrm{T}_{\mathrm{CM}}$ cells represent a limited size reservoir, which decreases at a very slow rate as a consequence of activation of antigen-induced responses, eliminating a fraction of infected cells. $\mathrm{T}_{\mathrm{TM}}$ cells represent the main reservoir in individuals where HAART treatment is started at a later stage. Here, a persistent proliferation of CD4+ $\mathrm{T}_{\mathrm{TM}}$ cells, although at low levels, is maintained by high amounts of interleukin 7 [33].

Another cellular sanctuary has been recently recognized in the CD4+ $\mathrm{T}_{\mathrm{SCM}}$ ( $\mathrm{T}$ memory stem cells). This long-term HIV-1 reservoir seems to be related to $\mathrm{T}_{\mathrm{SCM}}$ intrinsic properties, such as self-renewal, escape from apoptotic mechanisms and long time survival [34]. Cellular sanctuaries are also represented by monocytes, macrophages, naive CD4+ $\mathrm{T}$ cells, astrocytes, and follicular cells [3]. In particular, macrophages act as chronic and latent viral reservoirs in infected patients [35, 36], because the life span of HIV-1 infected cells is prolonged by sophisticated mechanisms adopted by the virus within these cells. Outstanding examples are the production of pro-survival factors, induced by the viral envelope glycoprotein, which downregulate the death receptor (tumor necrosis factor-related apoptosis-inducing ligand [TRAIL]-R1) [37], or the induction of the telomerase activity, which is known to contribute to cellular protection against oxidative stress [38].

\section{The P-glycoprotein: A Molecular Barrier to ARVs}

In addition to the anatomical barriers described above, the P-gp (P-glycoprotein) system represents a crucial molecular barrier to various ARVs. The P-glycoprotein is a drug efflux transporter which pumps drugs out of the cell, preventing their intracellular accumulation [39], and it has a central role in cellular detoxification not only during cytotoxic chemotherapy or antibiotics treatment, but also treatment with ARVs. Indeed, P-gp is a major cause for the reduced concentration of ARVs within cerebrospinal fluid and semen, since it is 
overexpressed in both BBB and BTB [39]. P-gp is also largely expressed in intestinal mucosa, thus representing a real obstacle in oral absorption of some ARVs such as saquinavir [40]. It has been recently demonstrated that HIV-1 exposure actively increases P-gp expression in bowel mucosa, decreasing the concentration of orally administered ARVs within the gut-associated lymphoid tissue [41]. In this context, it has been demonstrated that P-gp is overexpressed in the cervico-vaginal mucosa as well, which is the target of some recent studies on HIV-1 sexual transmission [42, 43]. Indeed, various pharmacological formulations of ARVs have been tested vaginally or orally for a pre-exposure prophylaxis of HIV-1 in women, but the presence of P-gp significantly diminishes the efficacy of the administered ARV [43]. Finally, P-gp can be highly overexpressed in response to therapy administration, as well documented by studies on CD8+ $\mathrm{T}$ lymphocytes isolated from the peripheral blood of HIV-1-infected patients under ARV treatment [44]. In conclusion, P-gp system plays a crucial role in preventing the penetration of ARVs into HS, and overcoming this barrier is of primary importance for proper viral eradication.

\section{Nano-delivery Systems to Catch the Anatomical HIV-1 Sanctuaries}

\subsection{Central Nervous System}

Overcoming of anatomical barriers, aimed to reach effective concentration of ARVs in specific HIV-1 reservoirs, has been widely investigated in the last decade by various nanotechnological approaches (Table 2). In this context, the most studied sanctuary

Table 2 Nanoparticles for the delivery of ARVs into HIV-1 sanctuaries.

\begin{tabular}{|c|c|c|c|}
\hline Sanctuary & Nanoparticle & $\begin{array}{l}\text { Delivered } \\
\text { ARV }\end{array}$ & Experimental model \\
\hline \multirow{7}{*}{ Central nervous systems } & quantum rods-Tf [48] & saquinavir & In vitro BBB model \\
\hline & RMP-7/MMA-SPM-NP [49] & $\begin{array}{l}\text { stavudine } \\
\text { saquinavir delavirdine }\end{array}$ & In vitro $\mathrm{BBB}$ model \\
\hline & TAT-NP [53] & ritonavir & In vitro $\mathrm{BBB}$ and in vivo model \\
\hline & $\begin{array}{l}\text { poly(ethylene oxide)-poly(propylene } \\
\text { oxide) micelle [54] }\end{array}$ & efavirenz & In vivo model \\
\hline & MYTS [55] & enfuvirtide & In vitro BBB and in vivo model \\
\hline & magnetic liposome [56] & AZTTP & In vitro BBB model \\
\hline & folate-nanoART [57] & $\begin{array}{l}\text { Atazanavir } \\
\text { ritonavir } \\
\text { indinavir } \\
\text { efavirenz } \\
\end{array}$ & In vitro $\mathrm{BBB}$ and in vivo model \\
\hline \multirow{6}{*}{ Lymphoid tissue } & anti-HLA-DR immunoliposome [59] & indinavir & In vivo model \\
\hline & lipid NP [60] & indinavir & In vivo model \\
\hline & PEG-lipid NP [61] & indinavir & In vivo model \\
\hline & Man-liposome [62] & zidovudine & In vivo model \\
\hline & PEG-elastic liposome [63] & zidovudine & In vivo model \\
\hline & folate-nanoART [64] & $\begin{array}{l}\text { ritonavir } \\
\text { atazanavir }\end{array}$ & In vivo model \\
\hline Male genital tract & PLGA [65] & $\begin{array}{l}\text { lopinavir } \\
\text { ritonavir } \\
\text { efavirenz }\end{array}$ & In vivo model \\
\hline \multirow{6}{*}{ Cellular sanctuaries } & anti-CD4- lipid NP [70] & indinavir & CD4+- cells \\
\hline & anti-CD4-immunoliposome [71] & saquinavir nevirapine & CD4+ Jurkat T cells \\
\hline & lipid NP- bryostatin-2 [72] & nelfinavir & CD4+ T-cells and in vivo model \\
\hline & glycine/mannose-PPI [73] & efavirenz & monocytes/macrophages \\
\hline & tuftsin-PPI [74] & efavirenz & macrophages \\
\hline & mannose-PPI [75] & lamivudine & macrophages \\
\hline
\end{tabular}


is definitely the CNS, since ability of nanoformulation to allow or increase permeation of ARVs across the BBB is a strongly appealing clinical feature. In 2008, $\mathrm{Xu}$ and colleagues designed a $\mathrm{Tf}$ (transferrin)-conjugated quantum rod for the specific targeting of BBB. This Tf-conjugated nanoparticle was able to cross an in vitro BBB model by exploiting a receptor-mediated transport [45]. Indeed, it is known that BBB endothelial cells overexpress TfR (transferrin receptor) [46], which acts as a transporter for therapeutic biomolecules to the brain [47]. The same nanoparticle was then employed for the conjugation of saquinavir, and in vitro studies have demonstrated that the trans-BBB permeation of the nanoformulated ARV was higher than that of the free drug. As a consequence, a more efficient antiretroviral activity on HIV-1 infected peripheral blood mononuclear cells was demonstrated [48]. Another targeted delivery of ARVs across the BBB has been obtained with MMA-SPM (methylmethacrylate-sulfopropylmethacrylate). nanoparticles functionalized with the bradykinin analog RMP-7, a receptor agonist of the bradykinin type II receptor which is primarily expressed in neuronal and vascular tissues.

These NPs loaded with stavudine, saquinavir or delavirdine have demonstrated enhanced permeation across the $\mathrm{BBB}$, exploiting both the transcellular transport of NPs as well as RMP-7-mediated increase of paracellular permeability [49].

Other nanoparticles have been designed as brain drug delivery systems by exploiting the penetration capability of molecules conjugated on their surface, such as cell penetrating peptides and analogues [50], or the peculiar chemical-physical properties of NP shell (i.e. pluronic block and PMA-based amphiphilic copolymers) [51, 52]. In 2008, Rao et al. demonstrated that TAT (trans-activator of transcription) peptide-conjugated nanoparticles were able to increase the CNS transport of the encapsulated ritonavir across the BBB in mouse [53]. Encapsulation of efavirenz into a poly (ethylene oxide)—poly(propylene oxide) micelle increased its bioavailability into the CNS upon intranasal administration [54]. In 2015, our group demonstrated that enfuvirtide conjugated to PMA-coated iron oxide NPs is allowed to cross BBB in vitro and in vivo. In this case, the nano-drug penetration into BBB endothelial cells was mediated by the absorption of the amphiphilic coating on cell membrane, and upon nanocomplex dissociation within the endosomal/lysosomal complex, enfuvirtide was released in brain parenchyma. ${ }^{[55]}$ Since enfuvirtide is a structurally complex molecule known to be unable to reach the cerebrospinal fluid in a detectable amount [15], hypothetically, this study may suggest a suitable system for the passage of most ARVs across the BBB.

Although liposomes are the most utilized drug carriers, their application as ARV delivery systems to the CNS is limited by inefficient loading of water-soluble drugs, low stability, and leakiness of loaded drug during storage [10]. Among several studies performed with liposomes [6], interesting results have been obtained with magnetic liposomal nanoformulations of azidothymidine 5'-triphosphate, which is the active form of azidothymidine. These magnetic liposomes can transmigrate across BBB in vitro, either directly or by a monocyte-mediated transport, under the influence of an external magnetic field [56].

As already mentioned, nanoARTs have also proved as valid tools for CNS penetration upon encapsulation in macrophages. Atazanavir, ritonavir, indinavir, and efavirenz, formulated as nanoART, and internalized into brain mononuclear phagocytes were efficiently translocated to human BBB endothelial cells by a cell-to-cell contact mechanism. Permeation into the CNS, especially that of folate-coated nanoart, was associated with decreased viral load and inflammation in the brain of HIV-1 infected mice [57].

\subsection{Lymphoid Tissue}

Targeting of lymphoid tissue with nanoformulated 
ARVs has been investigated since 1999 when the first liposomes directed towards HLA-DR-expressing monocyte/macrophages and activated $\mathrm{CD} 4+\mathrm{T}$ lymphocytes in this site were developed [58]. Three years later, it was demonstrated that the nanoformulation of indinavir in this NP was able to induce a 126-fold higher concentration of drug in the lymphoid tissue of injected mice, with a therapeutic window of at least 15 days post-injection [59]. In 2003, lipid NPs were used for the lymphatic delivery of indinavir in infected macaques, enhancing its peripheral and visceral lymph nodes concentration up to values 23-fold higher than in plasma and providing a significant virus load reduction both in lymphoid tissue and plasma [60]. This system was then optimized by saturation of indinavir lipid NPs with polyethylene glycol, obtaining a further enhancement of drug level in lymph nodes [61]. In 2008, zidovudine-loaded liposomes with different surface charges were exploited to improve its delivery to lymphoid virus reservoir, demonstrating that negatively charged liposomes had higher accumulation in lymph nodes compared to positively charged nanoART [62]. Moreover, a surface coating with mannose, whose receptors are highly expressed on immune cells, induced a much higher lymphatic accumulation. In the same year, the transdermal administration of PEGylated liposomal zidovudine formulation induced a 27-fold higher accumulation of zidovudine in lymphoid tissues compared to free drug, likely associated to a higher uptake in lymphoid cells [63]. More recently, a specialized folate-modified nanoART, containing ritonavir and atazanavir, was loaded in macrophages, thanks to folate receptor-mediated cellular uptake, thus obtaining an efficient and long-lasting delivery of ARVs in mice lymph nodes after a single administration [64].

\subsection{Male Genital Tract}

So far, no study about the permeation of nanoformulated ARVs across the BTB has been reported, with the only exception of a broad-spectrum research aimed to investigate the effect of nanocomplexation of ARVs on their distribution in different organs, including testes. The results of this study indicated that lopinavir, ritonavir and efavirenz administered into PLGA (Poly Lactic-co-Glycolic Acid) nanoparticles were able to accumulate in mice testes for up to 35 days [65], due to efficient entrapment, cellular uptake, retention and sustained release of drugs provided by these NPs [66]. Moreover, few other studies that investigated the biodistribution and systemic toxicity of iron, silver and gold nanocompounds administered to mice are available [67-69]. They describe a suitable distribution of these NPs in testes, thus suggesting a promising employment of inorganic nanoparticles for the delivery of ARVs into the male genital tract.

\section{Nano-delivery of ARVs to the Cellular Sanctuaries}

Most nanotechnological strategies for the delivery of ARVs to the cellular HIV-1 sanctuaries are based on the active targeting of the CD4 receptors (Table 2). CD4-targeted lipid NPs nanocomplexed with indinavir demonstrated a selective binding and uptake on CD4+-HIV-1 host cells, with an increased anti-HIV-1 effect. The hypothesized delivery mechanism involved the interaction of the nanoformulation with membrane CD4, followed by its endocytosis, and pH-dependent endosomal escape of indinavir into the cell cytoplasm, where it inhibits the viral protease [70]. Very recently, a stealth anti-CD4-targeted immunoliposome loaded with saquinavir and nevirapine has been developed which demonstrated an efficient uptake of the drugs into CD4+ Jurkat $\mathrm{T}$ cells and an enhanced anti-viral efficacy at significantly lower concentrations when compared to the free drug [71]. Kovochich and colleagues designed lipid nanoparticles loaded with bryostatin-2, a protein kinase C activator able to activate latent HIV-1 infections. These nanoformulations were demonstrated to target and 
activate CD4+ T-cells and stimulate latent virus production, both in vitro and in a humanized mouse model. The simultaneous incorporation of bryostatin-2 and the protease inhibitor nelfinavir gave rise to an optimized nanoformulated ARV that was able to both activate the latent virus expression and inhibit viral spread in HIV-1-infected T lymphoblastoid cells after 3 days of incubation [72].

Regarding macrophages reservoirs, nanoART has been demonstrated to efficiently translocate into these cells, thus exerting an increased anti-HIV-1 activity (see previous section) [8, 9]. Another model of Mo/Mac (monocytes/macrophages)-targeted nanoparticles was developed by Dutta and colleagues in 2007. They produced a 5th generation poly (propyleneimine) dendrimer (PPI)-based nanocontainer loaded with the antiretroviral efavirenz, and conjugated with glycine and mannose to optimize its targeted delivery, improving the release kinetic of the drug in comparison to the non-targeted NP and overcoming limitations imposed by NP toxicity. The best ARV cellular uptake was obtained with the mannose conjugated dendrimer due to its interaction with lectin receptors [73]. Another strategy to specifically deliver efavirenz in monocytes/macrophages has been exploited functionalizing the NP surface with tuftsin, a peptide known to specifically bind macrophages and activate several cell functions, such as phagocytosis. This tuftsin-conjugated PPI improved drug release with negligible cytotoxicity, enhanced cellular uptake within macrophages and enhanced drug efficacy [74]. Mannose-conjugated PPI was also loaded with lamivudine [75]. Higher ARV macrophages uptake and antiretroviral activity were observed with the lectin-targeted nanoformulations, as compared to the non-targeted dendrimer or to the free drug.

\section{Nanotechnological Approaches for P-gp Barrier Overcoming}

With regards to the P-gp barrier, nanostructured lipid carriers have been recently developed to enhance saquinavir bioavailability upon oral administration. Interestingly, these nanoparticles were able to overcome P-gp-mediated efflux from bowel mucosa, allowing caveolae- and clathrin-mediated transcytosis of the drug, with subsequent absorption [76]. Currently, some chemical strategies combining antiretroviral activity with inhibition of P-gp are being tested in preclinical experiments and have yielded encouraging results, mainly regarding penetration of ARVs through the BBB [77, 78]. However, although potentially significant, ARVs nanoformulations to specifically overcome P-gp activity still remain poorly investigated.

\section{Conclusions}

Nanotechnology has been exploited to enhance the pharmacokinetic properties of ARVs, and some efforts have been attempted to make HIV-1 therapy penetrate through selective biological barriers to reach HIV-1 replication sanctuaries. Preliminary results are more than encouraging. Certainly, further research is required to better define the antiretroviral activity once the nano-drug has passed through the barrier. Furthermore, the next step will be the multiple encapsulation of ARVs into a single nanoparticle for a smart contemporary targeting of HIV-1 replication and infection pathways. Combined together, nanoformulations containing multiple ARVs that are suited to cross physiological barriers could lead to the generation of a novel class of anti-HIV-1 drugs, with the possibility of a combined antiretroviral strategy for both anatomical and intracellular reservoirs of HIV-1. A prompt translation of preclinical results into clinical practice will hopefully revolutionize HIV-1 therapy in the next future.

\section{References}

[1] Sarmati, L., Parisi, S. G., Montano, M., Andreis, S., Scaggiante, R., Galgani, A., Viscione, M., Maffongelli, G., Ricciardi, A., Andreoni, C., Boros, S., Palù, G., and Andreoni, M. 2012. “Nevirapine Use, Prolonged 
Antiretroviral Therapy and High CD4 Nadir Values Are Strongly Correlated with Undetectable HIV-DNA and -RNA Levels and CD4 Cell Gain.” The Journal of Antimicrobial Chemotherapy 67 (12): 2932-8.

[2] Ostrowski, M., Benko, E., Yue, F.Y., Kim, C. J., Huibner, S., Lee, T., Singer, J., Pankovich, J., Laeyendecker, O., Kaul, R., Kandel, G., and Kovacs, C. 2015. "Intensifying Antiretroviral Therapy with Raltegravir and Maraviroc during Early Human Immunodeficiency Virus (HIV) Infection Does Not Accelerate HIV Reservoir Reduction.” Open Forum Infectious Diseases 2 (4): ofv138.

[3] Svicher, V., Ceccherini-Silberstein, F., Antinori, A., Aquaro, S., and Perno, C. F. 2014. "Understanding HIV Compartments and Reservoirs.” Current HIV/AIDS Reports 11 (2): 186-94.

[4] Schafer, V., von Briesen, H., Andreesen, R., Steffan, A. M., Royer, C., Tröster, S., Kreuter, J., and Rübsamen-Waigmann, H. 1992. "Phagocytosis of Nanoparticles by Human Immunodeficiency Virus (HIV)-Infected Macrophages: A Possibility for Antiviral Drug Targeting.” Pharmaceutical Research 9 (4): 541-6.

[5] Nowacek, A., and Gendelman, H. E. 2009. "NanoART, NeuroAIDS and CNS Drug Delivery.” Nanomedicine (Lond) 4 (5): 557-74.

[6] Edagwa, B. J., Zhou, T., McMillan, J. M., Liu, X., and Gendelman H. E. 2014. "Development of HIV Reservoir Targeted Long Acting Nanoformulated Antiretroviral Therapies.” Current Medicinal Chemistry 21 (36): 4186-98.

[7] Dash, P. K., Gendelman, H. E., Roy, U., Balkundi, S., Alnouti, Y., Mosley, R. L., Gelbard, H. A., McMillan, J., Gorantla, S., and Poluektova LY. 2012. "Long-Acting Nanoformulated Antiretroviral Therapy Elicits Potent Antiretroviral and Neuroprotective Responses in HIV-1-Infected Humanized Mice.” AIDS 26 (17): 2135-44.

[8] Nowacek, A. S., McMillan, J., Miller, R., Anderson, A., Rabinow, B., and Gendelman, H. E. 2010. "Nanoformulated Antiretroviral Combinations Extend Drug Release and Antiretroviral Responses in HIV-1 Infected Macrophages: Implications for NeuroAIDS Therapeutics.” Neuroimmune Pharmacology 5 (4): 592-601.

[9] Nowacek, A. S., Balkundi, S., McMillan, J., Roy, U., Martinez-Skinner, A., Mosley, R. L., Kanmogne, G., Kabanov, A. V., Bronich, T., and Gendelman, H. E. 2011. "Analyses of Nanoformulated Antiretroviral Drug Charge, Size, Shape and Content for Uptake, Drug Release and Antiviral Activities in Human Monocyte-Derived Macrophages." Journal of Control Release 150 (2): 204-11.
[10] Sagar, V., Pilakka-Kanthikeel, S., Pottathil R., Saxena, S. K., and Nair, M. 2014. Towards nanomedicines for neuroAIDS. Reviews in Medical Virology 24 (2): 103-24.

[11] Kramer-Hämmerle, S., Rothenaigner, I., Wolff, H., Bell, J. E., and Brack-Werner, R. 2005. "Cells of the Central Nervous System as Targets and Reservoirs of the Human Immunodeficiency Virus." Virus Research 111 (2): 194-213.

[12] Solas, C., Lafeuillade, A., Halfon, P., Chadapaud, S., Hittinger, G., and Lacarelle, B. 2003. "Discrepancies between Protease Inhibitor Concentrations and Viral Load in Reservoirs and Sanctuary Sites in Human Immunodeficiency Virus-Infected Patients.” Antimicrobial Agents and Chemotherapy 47 (1): 238-43.

[13] Mahajan, S. D., Roy, I., Xu, G., Yong, K. T., Ding, H., Aalinkeel, R., Reynolds, J., Sykes, D., Nair, B. B., Lin, E. Y., Prasad, P. N., and Schwartz, S. A. 2010. "Enhancing the Delivery of Antiretroviral Drug "Saquinavir" across the Blood Brain Barrier Using Nanoparticles.” Current HIV Research 8 (5): 396-404.

[14] Best, B. M., Letendre, S. L., Koopmans, P., Rossi, S. S., Clifford, D. B., Collier, A. C., Gelman, B. B., Marra, C. M., McArthur, J. C., McCutchan, J. A., Morgello, S., Simpson, D. M., Capparelli, E. V., and Ellis, R. J. 2012. "Low Cerebrospinal Fluid Concentrations of the Nucleotide HIV Reverse Transcriptase Inhibitor, Tenofovir." Journal of Acquired Immune Deficiency Syndromes 59 (4): 376-81.

[15] Price, R. W., Parham, R., Kroll, J. L., Wring, S. A., Baker, B., Sailstad, J., Hoh, R., Liegler, T., Spudich, S., Kuritzkes, D. R., and Deeks, S. G. 2008. "Enfuvirtide Cerebrospinal Fluid (CSF) Pharmacokinetics and Potential Use in Defining CSF HIV-1 Origin.” Antiviral Therapy 13 (3): 369-74.

[16] Hong, S., and Banks, W. A. 2015. "Role of the Immune System in HIV-associated Neuroinflammation and Neurocognitive Implications.” Brain Behavior and Immunity 5: 1-12.

[17] Watkins, C. C., and Treisman, G. J. 2015. "Cognitive Impairment in Patients with AIDS-Prevalence and Severity.” HIV AIDS (Auckl) 7: 35-47.

[18] Grant, I., Franklin, D. R., Deutsch, R., Woods, S. P., Vaida, F., Ellis, R. J., Letendre, S. L., Marcotte, T. D., Atkinson, J. H., Collier, A. C., Marra, C. M., Clifford, D. B., Gelman, B. B., McArthur, J. C., Morgello, S., Simpson, D. M., McCutchan, J. A., Abramson, I., Gamst, A., Fennema-Notestine, C., Smith, D. M., and Heaton, R. K. 2014. "Asymptomatic HIV-associated Neurocognitive Impairment Increases Risk for Symptomatic Decline.” Neurology 82: 2055-62.

[19] Valcour, V. G., Ananworanich, J., Agsalda, M., Sailasuta, N., Chalermchai, T., Schuetz, A., Shikuma, C., Liang, C. 
Y., Jirajariyavej, S., Sithinamsuwan, P., Tipsuk, S., Clifford, D. B., Paul, R., Fletcher, J. L., Marovich, M. A., Slike, B. M., DeGruttola, V., and Shiramizu, B. 2013. "HIV DNA Reservoir Increases Risk for Cognitive Disorders in cART-naïve Patients.” PLoS One 8 (7): e70164.

[20] Schrier, R. D., Hong S., Crescini M., Ellis R., Pérez-Santiago, J., Spina, C., and Letendre, S. 2015. "Cerebrospinal Fluid (CSF) CD8+ T-cells that Express Interferon-gamma Contribute to HIV Associated Neurocognitive Disorders (HAND).” PLoS One 10 (2): e0116526.

[21] Shiramizu, B., Williams, A. E., Shikuma, C., and Valcour, V. 2009. "Amount of HIV DNA in Peripheral Blood Is Proportional to the Severity of Neurocognitive Disorders.” The Journal of Neuropsychiatry and Clinical Neurosciences 21 (1): 68-74.

[22] Desplats, P., Dumaop, W., Smith, D., Adame, A., Everall, I., Letendre, S., Ellis, R., Cherner, M., Grant, I., and Masliah, E. 2013. "Molecular and Pathologic Insights from Latent HIV-1 Infection in the Human Brain.” Neurology 80 (15): 1415-23.

[23] Chen, M. F., Gill, A. J., and Kolson, D. L. 2014. "Neuropathogenesis of HIV-associated Neurocognitive Disorders: Roles for Immuneactivation, HIV Blipping and Viral Tropism.” Current opinion in HIV and AIDS 9 (6): 1-6.

[24] Gray, L. R., Roche, M., Flynn, J. K., Wesselingh, S. L., Gorry, P. R., and Churchill, M. J. 2014. "Is the Central Nervous System a Reservoir of HIV-1?” Current Opinion in HIV and AIDS 9 (6): 552-8.

[25] Cheng, C. Y., and Mruk, D. D. 2012 "The Blood-Testis Barrier and Its Implications for Male Contraception.” Pharmacological Reviews 64 (1): 16-64.

[26] Else, L. J., Taylor, S., Back, D. J., and Khoo, S. H. 2011. "Pharmacokinetics of Antiretroviral Drugs in Anatomical Sanctuary Sites: The Male and Female Genital Tract.” Antiviral Therapy 16 (8): 1149-67.

[27] Matusali, G., Dereuddre-Bosquet, N., Le Tortorec, A., Moreau, M., Satie, A. P., Mahé, D., Roumaud, P., Bourry, O., Sylla, N., Bernard-Stoecklin, S., Pruvost, A., Le Grand, R., and Dejucq-Rainsford, N. 2015. "Detection of Simian Immunodeficiency Virus in Semen, Urethra, and Male Reproductive Organs during Efficient Highly Active Antiretroviral Therapy.” Journal of Virology 89 (11): 5772-87.

[28] Robillard, K. R., Hoque, T., and Bendayan, R. 2012. "Expression of ATP-binding Cassette Membrane Transporters in Rodent and Human Sertoli Cells: Relevance to the Permeability of Antiretroviral Therapy at the Blood-Testis Barrier." The Journal of Pharmacology and Experimental Therapeutics 340 (1):
96-108.

[29] Cavert, W. Notermans, D. W., Staskus, K., Wietgrefe, S. W., Zupancic, M., Gebhard, K., Henry, K., Zhang, Z. Q., Mills, R., McDade, H., Schuwirth, C. M., Goudsmit, J., Danner, S. A., and Haase, A. T. 1997. "Kinetics of Response in Lymphoid Tissues to Antiretroviral Therapy of HIV-1 Infection.” Science 276 (5314): 960-4.

[30] Kinman, L., Brodie, S. J., Tsai, C. C., Bui, T., Larsen, K., Schmidt, A., Anderson, D., Morton, W. R., Hu, S. L., and Ho, R. J. 2003. "Lipid-Drug Association Enhanced HIV-1 Protease Inhibitor Indinavir Localization in Lymphoid Tissues and Viral Load Reduction: A Proof of Concept Study in HIV-2 287 -infected Macaques." J. Acquir Journal of Acquired Immune Deficiency Syndromes 34 (4): 387-97.

[31] Fletcher, C. V., Staskus, K., Wietgrefe, S. W., Rothenberger, M., Reilly, C., Chipman, J.G., Beilman, G.J., Khoruts, A., Thorkelson, A., Schmidt, T. E., Anderson, J., Perkey, K., Stevenson, M., Perelson, A. S., Douek, D. C., Haase, A. T., and Schacker, T. W. 2014. "Persistent HIV-1 Replication Is Associated with Lower Antiretroviral Drug Concentrations in Lymphatic Tissues." Proceedings of the National Academy of Sciences of the United States of America 111 (6): 2307-12.

[32] Horiike, M., Iwami, S., Kodama, M., Sato, A., Watanabe, Y., Yasui, M., Ishida, Y., Kobayashi, T., Miura, T., and Igarashi, T. 2009. "Lymph Nodes Harbor Viral Reservoirs that Cause Rebound of Plasma Viremia in SIV-Infected Macaques upon Cessation of Combined Antiretroviral Therapy.” Virology 423 (2): 107-18.

[33] Chomont, N., El-Far, M., Ancuta, P., Trautmann, L., Procopio, F. A., Yassine-Diab, B., Boucher, G., Boulassel, M. R., Ghattas, G., Brenchley, J. M,, Schacker, T. W., Hill, B. J., Douek, D. C., Routy, J. P., Haddad, E. K., and Sékaly, R. P. 2009. "HIV Reservoir Size and Persistence Are Driven by $\mathrm{T}$ Cell Survival and Homeostatic Proliferation.” Nature Medicine 15 (8): 893-900.

[34] Buzon, M., Sun, H., Li, C., Shaw, A., Seiss, K., Ouyang, Z., Martin-Gayo, E., Leng, J., Henrich, T. J., Li, J. Z., Pereyra, F., Zurakowski, R., Walker, B. D., Rosenberg, E. S., Yu, X. G., and Lichterfeld, M. 2014. "HIV-1 Persistence in CD4+ $\mathrm{T}$ Cells with Stem Cell-Like Properties.” Nature Medicine 20 (2): 139-44.

[35] Koppensteiner, H., Brack-Werner, R., and Schindler, M. 2012. "Macrophages and Their Relevance in Human Immunodeficiency Virus Type I Infection.” Retrovirology 9: 82-93.

[36] Kumar, A., and Herbein, G. 2014. "The Macrophage: A Therapeutic Target in HIV-1 Infection.” Molecular and Cellular Therapies 2: 10.

[37] Swingler, S., Mann, A. M., Zhou, J., Swingler, C., and 
Stevenson, M. 2007. “Apoptotic Killing of HIV-1-Infected Macrophages Is Subverted by the Viral Envelope Glycoprotein.” PLoS Pathogens 3 (9): 1281-90.

[38] Reynoso, R., Wieser, M., Ojeda, D., Bönisch, M., Kühnel, H., Bolcic, F., Quendler, H., Grillari, J., Grillari-Voglauer, R., and Quarleri, J. 2012. "HIV-1 induces telomerase activity in monocyte-derived macrophages - safeguarding one of its reservoirs?” Journal of Virology 86 (19): 10327-37.

[39] Robillard, K. R., Chan, G. N., Zhang, G., la Porte, C., Cameron, W., and Bendayan, R. 2014. "Role of P-glycoprotein in the Distribution of the HIV Protease Inhibitor Atazanavir in the Brain and Male Genital Tract.” Antimicrobial Agents and Chemotherapy 58 (3): 1713-22.

[40] Aungst, B. J. 1999. "P-glycoprotein, Secretory Transport, and Other Barriers to the Oral Delivery of Anti-HIV Drugs.” Advanced Drug Delivery Reviews. 39 (1-3): 105-16.

[41] Ellis, K., Marlin, J. W., Taylor, T. A., Fitting, S., Hauser, K. F., Rice, G., and McRae, M. 2015. "The Effects of Human Immunodeficiency Virus Infection on the Expression of the Drug Efflux Proteins P-glycoprotein and Breast Cancer Resistance Protein in a Human Intestine Model." The Journal of Pharmacy and Pharmacology 67 (2): 178-88.

[42] Zhou, T., Hu, M., Pearlman, A., Patton, D., and Rohan L. 2014. "Expression and Localization of p-glycoprotein, Multidrug Resistance Protein 4, and Breast Cancer Resistance Protein in the Female Lower Genital Tract of Human and Pigtailed Macaque.” AIDS Research and Human Retroviruses 30 (11): 1106-16.

[43] McGowan, I., Gomez, K., Bruder, K., Febo, I., Chen, B. A., Richardson, B. A., Husnik, M., Livant, E., Price, C., and Jacobson, C. 2011. "Phase 1 Randomized Trial of the Vaginal Safety and Acceptability of SPL7013 Gel (VivaGel) in Sexually Active Young Women (MTN-004).” AIDS 25 (8): 1057-64.

[44] Zhang, J. C., Xie, F., Yu, X. H., Deng, Z. Y., Wang, Y., Liang, P., Sun, L., and Zhang, F. X. 2014. "Expression Levels of P-glycoprotein in Peripheral Blood CD8+ T Lymphocytes from HIV-1-infected Patients on Antiretroviral Therapy.” International Journal of Molecular Medicine 33 (2): 431-40.

[45] Xu, G., Yong, K. T., Roy, I., Mahajan, S. D., Ding, H., Schwartz, S. A., and Prasad, P. N. 2008. "Bioconjugated Quantum Rods as Targeted Probes for Efficient Transmigration across an in vitro Blood-Brain Barrier.” Bioconjugate Chemistry 19 (6): 1179-85.

[46] Jefferies, W. A., Brandon, M. R., Hunt, S. V., Williams, A. F., Gatter, K. C., and Mason, D. Y. 1984. "Transferrin Receptor on Endothelium of Brain Capillaries.” Nature
312 (5990): 162-3.

[47] Jones, A. R., and Shusta, E. V. 2007. "Blood-Brain Barrier Transport of Therapeutics via Receptor-Mediation.” Pharmaceutical Research 24 (9): 1759-71.

[48] Mahajan, S. D., Roy I., Xu, G., Yong, K. T., Ding, H., Aalinkeel, R., Reynolds, J., Sykes, D., Nair, B. B., Lin, E. Y., Prasad, P. N., and Schwartz, S. A. 2010. "Enhancing the Delivery of Antiretroviral Drug "Saquinavir" across the Blood Brain Barrier Using Nanoparticles.” Current HIV Research 8 (5): 396-404.

[49] Kuo, Y. C., and Lee, C. L. 2012. "Methylmethacrylate-Sulfopropylmethacrylate

Nanoparticles with Surface RMP-7 for Targeting Delivery of Antiretroviral Drugs across the Blood-Brain Barrier." Colloids and surfaces. B, Biointerfaces 90: 75-82.

[50] Xia, H., Gao, X., Gu, G., Liu, Z., Hu, Q., Tu, Y., Song, Q., Yao, L., Pang, Z., Jiang, X., Chen, J., and Chen, H. 2012. "Penetratin-Functionalized PEG-PLA Nanoparticles for Brain Drug Delivery.” International Journal of Pharmaceutics 436 (1-2): 840-50.

[51] Kabanov, A. V., Li, S., Vinogradov, S. V., Alakhov, V. Y., Miller, D. W., and Kabanov, A. V. 2001. "Mechanism of Pluronic Effect on P-glycoprotein Efflux System in Blood-Brain Barrier: Contributions of Energy Depletion and Membrane Fluidization." The Journal of Pharmacology and Experimental Therapeutics 299 (2): 483-93.

[52] Hemmelman, M., Knoth, C., Schmitt, U., Allmeroth, M., Moderegger, D., Barz, M., Koynov, K., Hiemke, C., Rösch, F., and Zentel, R. 2011. "HPMA Based Amphiphilic Copolymers Mediate Central Nervous Effects of Domperidone.” Macromolecular Rapid Communications 32 (9-10): 712-7.

[53] Rao, K. S., Reddy, M. K., Horning, J. L., and Labhasetwar, V. 2008. "TAT-Conjugated Nanoparticles for the CNS Delivery of Anti-HIV Drugs.” Biomaterials 29 (33): 4429-38.

[54] Chiappetta, D. A., Hocht, C., Opezzo, J. A., and Sosnik, A. 2013. "Intranasal Administration of Antiretroviral-Loaded Micelles for Anatomical Targeting to the Brain in HIV." Nanomedicine 8 (2): 223-37.

[55] Fiandra, L., Colombo, M., Mazzucchelli, S., Truffi, M., Santini, B., Allevi, R., Nebuloni, M., Capetti, A., Rizzardini, G., Prosperi, D., and Corsi, F. 2015. "Nanoformulation of Antiretroviral Drugs Enhances Their Penetration across the Blood Brain Barrier in Mice.” Nanomedicine: Nanotechnology, Biology, and Medicine. 11 (6): 1387-97.

[56] Saiyed, Z. M., Gandhi, N. H., and Nair, M. P. 2010. "Magnetic Nanoformulation of Azidothymidine 
5'-triphosphate for Targeted Delivery across the Blood-Brain Barrier.” International Journal of Nanomedicine 5: 157-66.

[57] Kanmogne, G., Singh, S., Roy, U., Liu, X., McMillan, J., Gorantla, S., Balkundi, S., Smith, N., Alnouti, Y., Gautam, N., Zhou, Y., Poluektova, L., Kabanov, A., Bronich, T., and Gendelman, H. E. 2012. "Mononuclear Phagocyte Intercellular Crosstalk Facilitates Transmission of Cell-Targeted Nanoformulated Antiretroviral Drugs to Human Brain Endothelial Cells.” International Journal of Nanomedicine 7: 2373-88.

[58] Dufresne, I., Désormeaux, A., Bestman-Smith, J., Gourde, P., Tremblay, M. J., and Bergeron, M. G. 1999. "Targeting Lymph Nodes with Liposomes Bearing Anti-HLA-DR Fab' Fragments.” Biochimica et Biophysica Acta 1421 (2): 284-94.

[59] Gagne, J. F., Désormeaux, A., Perron, S., Tremblay, M. J., and Bergeron, M. G. 2002. "Targeted Delivery of Indinavir to HIV-1 Primary Reservoirs with Immunoliposomes.” Biochimica et Biophysica Acta 1558 (2): 198-210.

[60] Kinman, L., Brodie, S. J., Tsai, C. C., Bui, T., Larsen, K., Schmidt, A., Anderson, D., Morton, W. R., Hu, S. L., and Ho, R. J. 2003. "Lipid-Drug Association Enhanced HIV-1 Protease Inhibitor Indinavir Localization in Lymphoid Tissues and Viral Load Reduction: A Proof of Concept Study in HIV-2287-Infected Macaques.” Journal of Acquired Immune Deficiency Syndromes 34 (4): 387-97.

[61] Kinman, L., Bui, T., Larsen, K., Tsai, C. C., Anderson, D., Morton, W. R., Hu, S. L., and Ho, R. J. 2006. "Optimization of Lipid-Indinavir Complexes for Localization in Lymphoid Tissues of HIV-Infected Macaques.” Journal of Acquired Immune Deficiency Syndromes 42 (2): 155-61.

[62] Kaur, C. D., Nahar, M., and Jain, N. K. 2008. "Lymphatic Targeting of Zidovudine Using Surface-Engineered Liposomes.” Journal of Drug Targeting 16 (10): 798-805.

[63] Jain, S., Tiwary, A. K., and Jain, N. K. 2008. "PEGylated Elastic Liposomal Formulation for Lymphatic Targeting of Zidovudine.” Current Drug Delivery 5 (4): 275-81.

[64] Puligujja, P., McMillan, J., Kendrick, L., Li, T., Balkundi, S., Smith, N., Veerubhotla, R. S., Edagwa, B. J., Kabanov, A. V., Bronich, T., Gendelman, H. E., and Liu, X. M. 2013. "Macrophage Folate Receptor-Targeted Antiretroviral Therapy Facilitates Drug Entry, Retention, Antiretroviral Activities and Biodistribution for Reduction of Human Immunodeficiency Virus Infections.” Nanomedicine: Nanotechnology, Biology, and Medicine 9 (8): 1263-73.
[65] Destache, C. J., Belgum, T., Goede, M., Shibata, A., and Belshan, M.A. 2010. "Antiretroviral Release from Poly(DL-lactide-co-glycolide) Nanoparticles in Mice.” The Journal of Antimicrobial Chemotherapy 65 (10): 2183-87.

[66] Mandal, S., Zhou, Y., Shibata, A., and Destache, C. J. 2015. “Confocal Fluorescence Microscopy: An Ultra-sensitive Tool Used to Evaluate Intracellular Antiretroviral Nano-drug Delivery in HeLa Cells.” American Institute of Physics Advances 5 (8): 084803.

[67] Lankveld, D. P. K., Oomen, A. G., Krystek, P., Neigh, A., Troost-de Jong, A., Noorlander, C. W., Van Eijkeren, J. C., Geertsma, R. E., and De Jong, W. H. 2010. "The Kinetics of the Tissue Distribution of Silver Nanoparticles of Different Sizes.” Biomaterials 31 (32): 8350-61.

[68] Balasubramanian, S. K., Jittiwat, J., Manikandan, J., Ong, C. N., Yu, L. E., and Ong, W. Y. 2010. "Biodistribution of Gold Nanoparticles and Gene Expression Changes in the Liver and Spleen after Intravenous Administration in Rats.” Biomaterials 31 (8): 2034-42.

[69] Hue, J. J., Lee, H. J., Jon, S., Nam, S. Y., Yun, Y. W., Kim, J. S., and Lee, B. J. 2013. "Distribution and Accumulation of Cy5.5-labeled Thermally Cross-Linked Superparamagnetic Iron Oxide Nanoparticles in the Tissues of ICR Mice.” Journal of Veterinary Science 14 (4): 473-9.

[70] Endsley, A. N., and Ho, R. J. Y. 2012. "Enhanced Anti-HIV Efficacy of Indinavir after Inclusion in CD4 Targeted Lipid Nanoparticles.” Journal of Acquired Immune Deficiency Syndromes 61 (4): 417-24.

[71] Ramana, L. N., Sharma, S., Sethuraman, S., Ranga, U., and Krishnan, U. M. 2015. "Stealth Anti-CD4 Conjugated Immunoliposomes with Dual Antiretroviral Drugs-Modern Trojan Horses to Combat HIV.” European Journal of Pharmaceutics and Biopharmaceutics 89: 300-11.

[72] Kovochich, M., Marsden, M. D., and Zack, J. A. 2011. "Activation of Latent HIV Using Drug-Loaded Nanoparticles.” PLoS One 6(4): e18270.

[73] Dutta, T., Agashe, H. B., Garg, M., Balakrishnan, P., Kabra, M., and Jain, N. K. 2007. "Poly (propyleneimine) Dendrimer Based Nanocontainers for Targeting of Efavirenz to Human Monocytes/Macrophages in vitro." Journal of Drug Targeting 15(1): 89-98.

[74] Dutta, T. Garg, M., and Jain, N. K. 2008. "Targeting of Efavirenz Loaded Tuftsin Conjugated Poly(propyleneimine) Dendrimers to HIV Infected Macrophages in vitro." European Journal of Pharmaceutical Sciences 34 (2-3): 181-9.

[75] Dutta, T., and Narendra, K. 2007. “Targeting Potential and Anti-HIV Activity of Lamivudine Loaded 
Mannosylated poly (propyleneimine) Dendrimer.” Biochimica et Biophysica Acta 1770 (4): 681-6.

[76] Beloqui, A., Solinís, M. Á., Gascón, A. R., del Pozo-Rodríguez, A., des Rieux, A., and Préat, V. 2013. "Mechanism of Transport of Saquinavir-Loaded Nanostructured Lipid Carriers across the Intestinal Barrier.” Journal of Controlled Release 166 (2): 115-23.

[77] Namanja, H. A., Emmert, D., Davis, D. A., Campos, C.,
Miller, D. S., Hrycyna, C. A., and Chmielewski, J. 2012. "Toward Eradicating HIV Reservoirs in the Brain: Inhibiting P-glycoprotein at the Blood-Brain Barrier with Prodrug Abacavir Dimers." Journal of the American Chemical Society 134 (6): 2976-80.

[78] Chmielewski, J., and Hrycyna, C. 2012. "Tools for Eradicating HIV in the Brain: Prodrug Dimeric Inhibitors of P-gp.” Therapeutic Delivery 3 (6): 689-92. 